\title{
Nano-Games for Cultural Venues: the HEAL game
}

\author{
Rompa Jenny $^{1}$, Lepouras George ${ }^{1}$, Antoniou Angela ${ }^{1}$, Pequenao Joao ${ }^{2}$ \\ ${ }^{1}$ HCI-VR Lab, Department of Informatics and Telecommunications, University of the \\ Peloponnese \\ $\{j r$, gl,angelant $\} @ u o p . g r$ \\ ${ }^{2}$ CERN, joao.pequenao@cern.ch
}

\begin{abstract}
Many are the times when visitors leave an exhibition without a clue about its message. In an effort to eliminate this phenomenon, museums, institutes and organisations (MIOs) adopt more attractive, engaging and fun content to provide a high quality and fast learning experience. Games seem to be an interesting suggestion to this approach but they are time consuming. To this end, we introduce nano-games. We define nano-games as short, easy to master, self-contained games of a single level of difficulty, having basic and direct rules that stay unaltered throughout the play and challenge players with clearly defined goals reachable within tens of seconds of gameplay. This approach has been adopted and evaluated in the premises of European Center for Nuclear Research (CERN).
\end{abstract}

Keywords: digital games, nano-games, CERN, HEAL

\section{Introduction}

Museums started with an initial aim to collect, preserve, protect and display objects, specimens and art. Throughout the years museums have evolved into educational, accessible, and innovative institutes serving different people with different needs and interests [1]. Nowadays, in addition to museums, there is also a high number of other organisations and institutes hosting their own exhibitions in their venues. Universities, sport venues, even monuments (as for example the Eiffel Tower), can have or host exhibitions in order for their visitors to get to know more about the history hidden behind them and their contribution to our society. However, even though museums, institutes and organisations (MIOs) make an effort to ensure that every visitor leaves their venues with an understanding of what they wished to communicate, often visitors leave without a clue about what the exhibition was about [2]. As a result, visitors are not willing to re-visit or recommend their experience to others.

In an effort to eliminate this phenomenon, MIOs have adopted a more visitor-centred approach aiming to offer more of an experience than just a visit. Exhibitions get enriched with more attractive, engaging and even fun content in order to not only captivate their visitors' interest but also provide a both high quality and fast learning experience, regardless of their audience's background and age. During the last years, games and especially educational games have proved to be a great means of knowledge acquisition and the way to go in achieving this visitor-centred learning environment [3, 4]. As a matter of fact, increasingly more cultural places [5-7] adopt educational games in their exhibitions in an effort to improve users' experience and enhance the learning outcome. Even though games seem to be a viable solution to a lasting challenge, one may argue that 
there are numerous constraints that make adopting games in MIOs more difficult than one may think. For example, in order for a user to be able to play a game, one needs to at least understand its gameplay and know how to operate its controls beforehand, meaning that a MIO visitor would need to spend more time than initially estimated for a simple visit. Mastering the game could take even longer as the user would have to be familiar with the gameplay in order to achieve its goal. Several studies have shown that MIO visitors spend no more than 90 minutes in exhibitions, regardless of their topic or size [8-14], and at most 2 minutes $[15,16]$ in front of an exhibit, meaning that one should understand the exhibit's content and purpose in a matter of seconds. To overcome the time constraint a new approach is essential.

Games can be exploited in different ways. Since visit time constrains the visitor's experience while in the MIO, one can exploit time before and/or after the visit to prolong the total duration and offer opportunities for new types of experience. The experience can be extended before the actual visit to the MIO while the visitor prepares her trip, as well as after as the visitor shares the experience with other people. To this end, games can be employed before, during, or after the users' visit. Each option offers different benefits and can be used in different cases. For example, in the first option, we proposed games to be played before the users' visit to the venue's exhibition $[17,18]$. The games in that case reveal visitors' visiting styles and cognitive preferences enabling personalised routing and tailored exhibit descriptions during their venue visit. They could thus, have a better experience during their visit. Neto et al. [19], present another approach where museum visitors were asked to play a knowledge game after a museum visit as an effort to increase visitors' learning on the museum's material and to test the knowledge acquired during the museum visit, while [20], presents an approach which encourages its users to share their MIO experience with their friends on Social Networks during and after their visit. With this approach the visitors' interest could be kept alive even after the museum visit.

However, there are cases it may not be feasible for visitors to play the game before or after their visit. In such cases, games can be put to use during one's visit in order to enhance their MIO experience. Although this seems like a promising way to go, adopting games in the manner we know it, can lead us to the same dead-end as before. This could happen due to the fact that games in general take time in order for someone to learn how to play as well as be able to understand the gameplay, regardless of whether or not achieving the game's goal. As a result, a different approach that minimises the total duration of a game play, while focusing on the specific messages a MIO wants to pass to its visitors, is necessary.

Micro-games have been explored by other researchers [21-24] and can offer a solution to the time constrain since they are relatively simple and do not require special skills to play while challenging their players with clearly defined goals reachable within minutes of game-play. In a study [22] that took place at the cities of Prague and Kladno in the Czech Republic, the mean time of user interaction with the game was 20 minutes. While this seems promising for museums, considering a single game an exhibit when placed in a MIOs exhibition and having in mind the total time spent by visitors in exhibitions, as mentioned above, one can straightaway realise that if a micro-game approach is adopted, then there is a high risk of visitors not giving the expected attention to the rest of the exhibits, especially if more than one micro-games are in place. In addition to that, the number of visitors tends to increase when the size of MIO is larger, meaning that more visitors will need to spend time interacting with a single exhibit or game. This gets even more difficult when groups of visitors enter the MIO exhibition at the same time. In these cases a solution would be to offer games that enhance the visitors' experience and communicate some basic message, but do not require much available time to play and even less time and skills to learn how to play.

To this end, we introduce the concept of nano-games. We define nano-games as short, easy to master, self-contained games of a single level of difficulty, having basic and direct rules that stay unaltered throughout the play and challenge players with clearly defined goals reachable within tens of seconds of gameplay. Nano-games can employ 
human gestures and postures in an effort to maximise the easiness and effortlessness of skills needed, and minimise both the practice time and the number of skills needed before playing the game. While games in general need at least some skills to be mastered, nanogames do not assume the availability of time to train the players. To this end, a nanogame can exploit the mechanics of well-known and popular games to reduce training period. This approach can make a noteworthy difference in the total time committed to play the game. For example, while micro-games can last minutes or tens of minutes as users first need to be introduced to the game and practice in order to be able to play and master the game, nano-games should last tens of seconds or a couple of minutes at most. This fact implies that nano-games should be mastered in a matter of seconds, without any need of practice or lengthy introduction. Moreover, while many games are multilevel, with an increasing difficulty, and require for their players to keep up with new rules (e.g. new obstacles or new capabilities), nano-games are single-level with unaltered rules keeping the "how to play" learning need to the minimum. In addition to the above, nanogames are targeted on specific concepts of utmost importance. By focusing only on passing a specific message, unnecessary details are prevented from catching users' attention and time. As a result, users can receive the main message of a nano-game in a matter of tens of seconds.

In the next pages of this paper, we present two examples of nano-games and we describe a case study that took place in an international organisation that exploits nanogames in its exhibition. Finally, we discuss the outcomes of the case studies.

\section{Case study: nano games for CERN}

The European Center for Nuclear Research (CERN) is an international research facility of great importance. Having a great history in the field of particle physics, CERN has gained increasing interest during the past few decades (Travellers' Choice for 2018 as voted in Tripadvisor [25]) from scholars, researchers and tourists. More than 110,000 people per year [26] visit CERN in an effort to learn more about the organisation but also about particle physics. In order to provide its visitors with the information they are seeking for, several exhibits are available to the public. The exhibits are both conventional (posters, photos, text), and interactive (tablets, interactive screens, etc.).

In most cases, CERN needs to provide its visitors with information related to its experiments. Some examples include the experiments' workflow, objectives or even results that the organization wishes to communicate to the public. In such circumstances, the need of developing a new nano-game occurs. To effectively approach the topic, brainstorming involving experts from diverse domains was essential. The brainstorming team included people that were able to identify and summarize the information the MIO needed to pass to its visitors, (b) came up with suggestions on how to approach the concept, and (c) executed the decided approach. The key challenge of the brainstorming team was to decide on the "main message" that nano-game needed to pass to players. This message had to be comprehensive enough to be described in a single sentence. Secondary messages could be listed and included in the final design solution as minor game elements. While each case and game is different, the main guideline was that when designing a nano-game one has to keep in mind that the nano-game should be based on an easily understood metaphor, while maintaining its fun aspect and shortness of game play.

Taking all the above into account, the CERN Media Lab team developed the LHC Interactive Tunnel, or else LIT, as part of CERN's exhibition. CERN Media Lab [27] designs and implements systems, software and content for Science Visualization and Communication in order to support CERN's education programmes. LIT is an immersive, interactive space implementing the CERN experience using everyday life metaphors. LIT hosted two interactive games, one of which is called Proton Football. Proton Football was developed in 2011 to make visitors experience physics, instead of reading or hearing 
pag. 6

about it using conventional ways. To play the game, users need to use their body parts for the gameplay to proceed. Proton Football was an early attempt at reducing the gameplay time, while conveying a clear message.

\subsection{Proton football}

Proton Football is a playful way to explain to CERN visitors how particles are accelerated in the LHC and what happens when two protons collide. To achieve that, the architecture of one of CERN's experiments (ATLAS) is shown on a video wall (which we will call "Wall" for the ease-of-use). Both players are given virtual protons, projected on the floor, to which they have to give energy by kicking and make them collide in order for the experiment to operate and produce data. Figures 1 and 2 show the Proton Football interface.

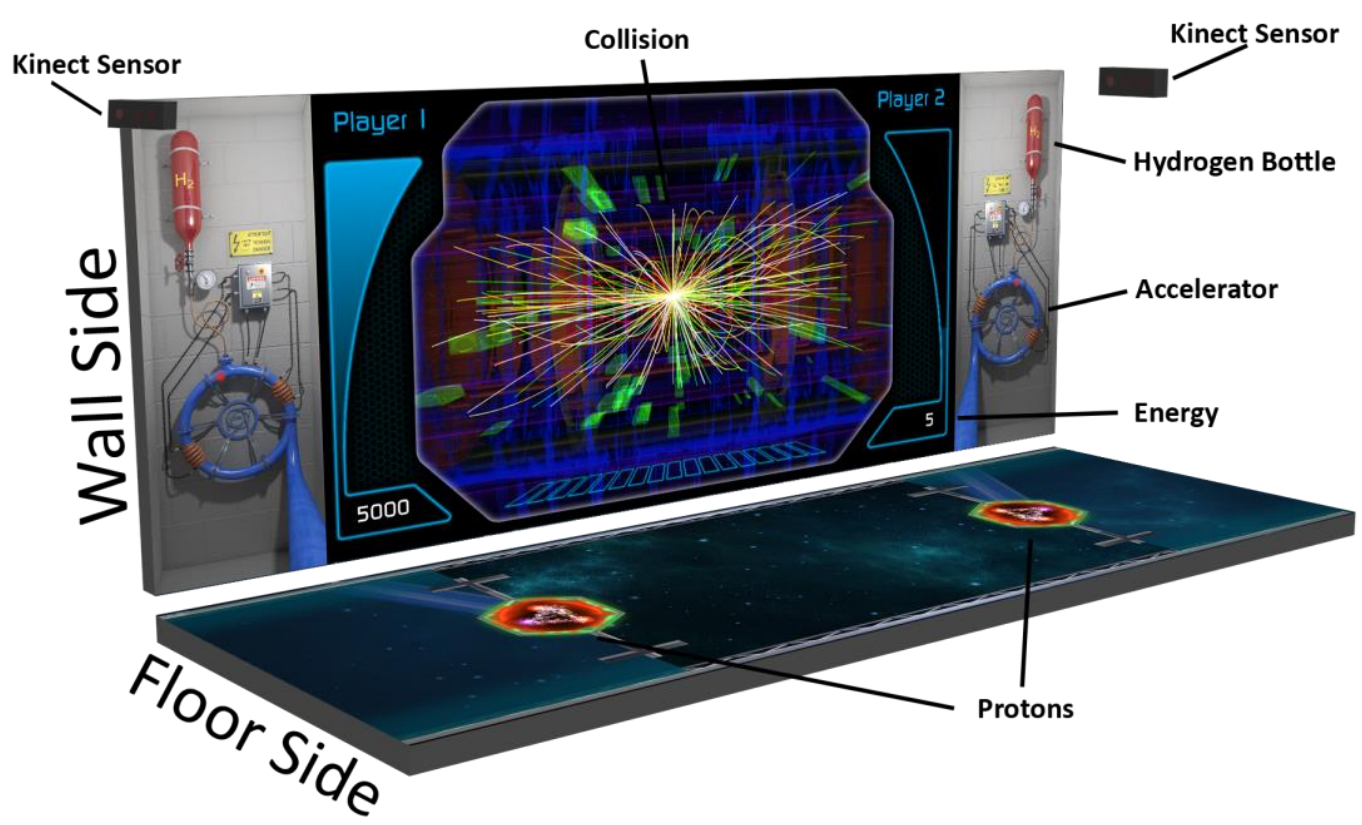

Figure 1. The Proton Football interface along with explanative captions on its components.

During the game, two users are asked to use their legs to physically kick the virtual protons positioned on the "Floor" side and make them collide. Protons are emitted from a Hydrogen bottle, located on the "Wall" side, get accelerated in the LHC and then are positioned in front of each player on the "Floor" side. To kick a virtual proton, the player has to do a physical strike to the proton in front of her, using one of her feet. The more intense the players' kicks are, the more energy is given to their protons. To achieve a collision, players must aim for their playmates' proton. In the event of a collision, the tracks of the new particles generated from the collision will be shown in both the "Wall" and the "Floor" sides. Tracks are distinguished by colour to achieve a visual categorization of the tracks depending on the momentum of the particles that created them. The bigger the collision, the more particles are produced.

Proton Football uses the mechanics of real life football. This approach was chosen by the Media Lab team in an effort to make the game suitable to be played by all visitors, regardless of their sex, age, origin, abilities, etc. Moreover, using a gesture that the majority of the visitors have done at some point in their lives (by kicking a ball) made the game seem more familiar.

While making the two protons collide may seem easy to accomplish, the two players need to coordinate in time, effort (kick's force) and direction in order to produce the best results. This constrain makes Proton Football not only more challenging but also closer to reality. This is because colliding particles in real life is not a trivial procedure and the coordination of multiple expert teams is essential for the experiment to be executed. 


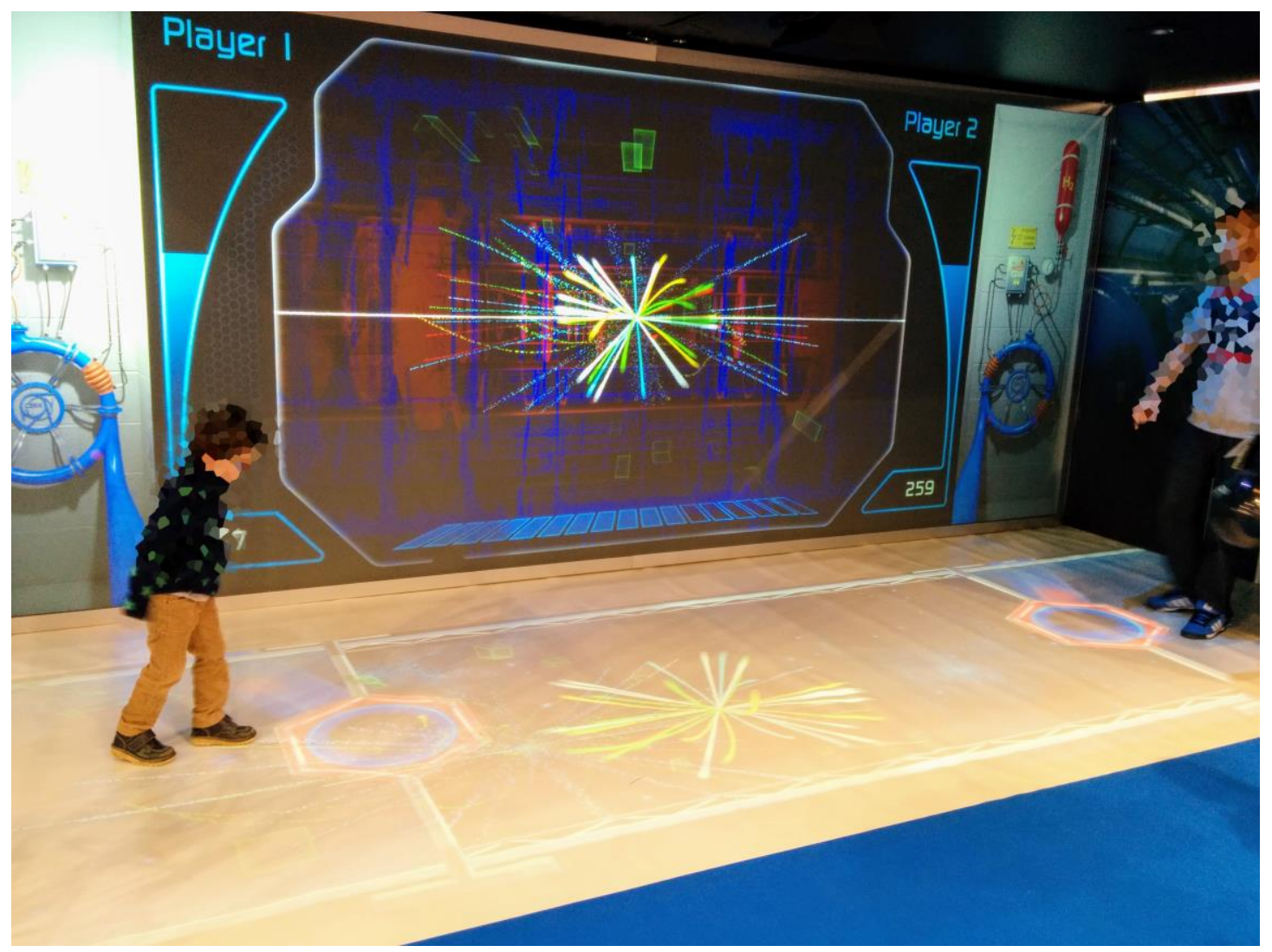

Figure 2. Users playing Proton Football in LIT immersive space, kicking from both sides; the proton energy and collision outcome is displayed on the Wall.

In order for someone to be ready to play, simple-to-follow instructions are presented by a human guide making it really easy to not only learn to play but also accomplish the game's goals. Completing a full gameplay scenario can even take down to 30 seconds.

Since Proton Football satisfies the definition of a nano-game, we believe that it is an ideal candidate for a first-level evaluation regarding nano-games used throughout visitors' MIO visits. A big portion of CERN visitors is high school students and under-informed people trying to find out more information about CERN and figure out what its usefulness to the community. When leaving Proton Football's immersive space, visitors seem to have an understanding of how a CERN experiment works and the basic physics of transforming energy into matter, through a fun medium. Moreover, the game coped under the pressure of loads of visitors' demands, of various ages and backgrounds from all over the world. Visitors were to be able to play, even though they were often part of a group visit where the time constrains get stricter.

\subsubsection{Assessment}

In order to find the strong points of Proton Football as well as its weaknesses, we conducted a first-level evaluation adopting the note-taking observational protocol [28] over the course of one year. With this approach we observed users' behaviour in real-life situations where it is more likely to record the frustrations and benefits than in case of a lab-based experiment following a set of instructions. Moreover, since we were not interfering with the users, they did not have to devote time into experience recording, meaning that there was more time for them to play and experience the game. Using this method also helps with product improvement as it is not limited to a set of questions hence it is more open to elaborate and get ideas. Even though many may argue that this type of observation is difficult to be replicable, we have repeatedly experienced users 
having encountered the same frustrations and benefits. We believe that this is due to the really high number of visitors the game has.

The evaluation took place at CERN's exhibition space called Microcosm, in Geneva, Switzerland. Users were free to play and talk to the guide(s) as much as they would like, including asking questions. During all this time, we recorded bugs and errors, as well as cases when the users could not understand the designer's intended mode of interaction. After a two-month observation period, we noticed patterns in user behaviours.

After conducting a first-level evaluation, we observed that visitors were generally really happy about Proton Football. First of all, they were excited that they could learn in such an immersive and interactive space. Parents were enthusiastic about their kids being able to play the games but also getting a vague idea of what protons, accelerators, experiments, etc, are. A lot of them claimed that it is really difficult to explain to children such complex concepts when they had no background information and admitted that Proton Football helped in comprehending the basic idea behind CERN. Elder visitors were also enthusiastic about being able to take part in such a «high-tech» game without ever having played electronic-games.

However, we identified a couple of issues during our observations. The first and most important one was that the users' experience was depended on the guide's approach and background knowledge. Since Proton Football had no embedded instructions, the presence of a guide was essential for multiple reasons. As most of the visitors had never visited CERN before or played with Proton Football, guides had to (a) explain its gameplay and components as well as (b) express the main idea behind it, clarifying some basic concepts related to CERN and (c) point out the correlation between the game graphics and the main idea. For example, there were cases when the interaction model failed as users would step on the virtual proton and expect for something to happen. Instead, they would need to stand one step behind and physically kick the virtual proton. In such cases, the guides' assistance was mandatory. Another example proving the necessity of guides was when the immersive space was left un-guided, as visitors that would approach it, would try to figure out how it works, but after a minute or two they would give up. In addition to the above, Proton Football was using the first generation of Microsoft Kinect [29] sensors, meaning that there were many occasions when users were not recognised by the sensors due to clothing misinterpretations. Guides' assistance was important in this case as well since they would explain to the users what they could do to alleviate the issue and be able to play. Another source of disappointment was that it is a two-player game. While it was more exciting to play with a friend, there were often times when single visitors would come to play and there was no other visitor available, apart from the guide. We have also observed that some users had difficulties trying to control both the energy and the direction in one gesture. Instead of changing the speed of their kick to achieve the best collision result, they kept at the same pace until they left the space. In other cases, the two players would kick the protons in different directions, never managing to collide them. Finally, there were also cases were users focused more on having fun, than actually getting some knowledge out of the game. We believe that part of this problem were the graphics, since players had to correlate information shown on both the back and floor screens. For example, users would often not notice that the protons were emitted from the hydrogen bottle on the "Wall" side, accelerated in the LHC and then positioned in front of them on the "Floor" side. As a result, users missed the information of where do protons come from. Such cases were also a reason that a human guide was essential. These findings were taken into consideration while designing a new game to promote a therapy based on CERN's technology.

\subsection{HEAL game}

Having in mind the above findings, we designed and developed an interactive game using similar philosophy and technologies. HEAL is a nano-game developed for LIT and intending to (a) inform visitors about Hadron Therapy and how it works, as well as (b) 
highlight CERN's involvement in other research fields, apart from physics and computer science, underlining its impact on our everyday lives through technological achievements. The game was designed with the following goals. After playing the game, users should be able to understand that (a) Hadron Therapy is more accurate than conventional therapy and (b) CERN relates to everyday life technologies.

In HEAL, users are challenged to destroy multiple cancer cells located in a humanoids' brain. The interface and interaction model underwent a few prototypes and tests. Figure 3 shows HEAL's first production interface.

Before entering HEAL's immersive space, users would see a message on the "Wall" side encouraging them to step to the footprints shown on the "Floor" side (Figure 4a). As soon as they would step in, some of HEAL's graphics (how-to-play instructions and hadron beam's direction preview line) would become visible.

During HEAL's gameplay, on the "Wall" side one may see a humanoids' brain along with some brownish blobs that represent the cancer cells. On the left side of the "Wall" users can see the energy bar along with some optical guidance (images) on how to interact with the game controllers that is always present. On the right side of the "Wall", a small info-box is always present to the users providing basic information for Hadron therapy. On the "Floor" side, users can see two footprints, indicating the place where they should stand, along with some arrows showing the direction to which they should kick. The rest of the "Floor" side is covered by a miniature of an accelerator, ending up to the "Wall" side. As soon as the users would kick, a virtual hadron beam would be emitted, accelerated and directed to the cancer cells.

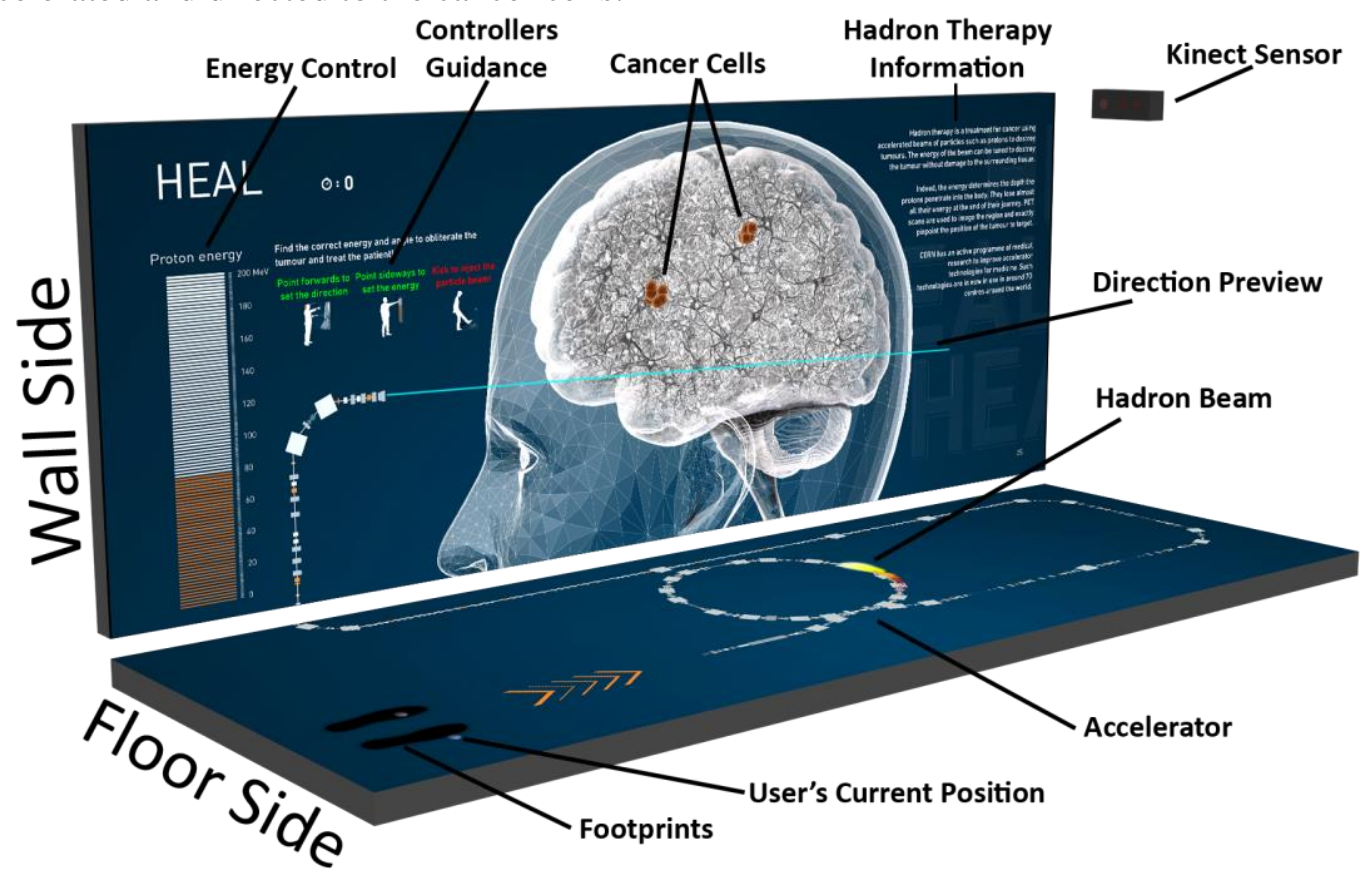

Figure 3. The HEAL interface along with explanative captions on its components.

Regarding the controls, visitors can use their hands to select the amount of energy to irradiate the cancer cells and then the emission direction, in contrast to Proton Football, where everything is controlled by a single gesture. In HEAL one has to just raise one of their hands in order to activate the control (Figure 5). To control the amount of energy, users have to raise their hand sideways pointing to the "Wall", while to control the direction, users have to raise their hand in front of them in parallel to the "Wall". While playing, they can either use one hand to control the energy and direction values one after another, or raise both of their hands at the same time and adjust the values simultaneously. Each control will "lock" to the desired value as soon as the user keeps her hand still for a couple of seconds. When a value was "locked", its corresponding instruction image on the 
pag. 10

"Wall" side would turn green (Figure 4b). Then, they may return their hand(s) close to their body.

To accelerate the hadron beam, users need to use their legs to physically kick the virtual hadrons that are going to be irradiated with the energy and to the direction they have already chosen. The beam will be first be accelerated in the miniature accelerator visible on the "Floor" side (Figure 4c) and then will travel to the "Wall" side, penetrating the humanoid's brain following the direction as set by the user (Figure 4d). The more the energy selected by the user, the further away the hadron beam will reach. To vanish the cancer cells, users need to select the exact amount of energy in order for the hadron beam to stop in the area where they are located. In case the energy is less or greater than the required one, the cancer cells will remain intact.

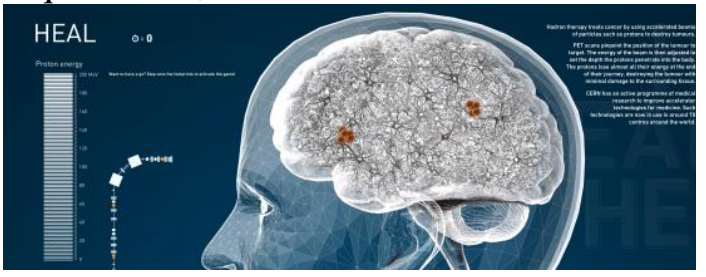

Figure 4a. The "Wall" interface before someone stepped on the footprints on the "Floor" side. On the right side, general information on Hadron Therapy are displayed.
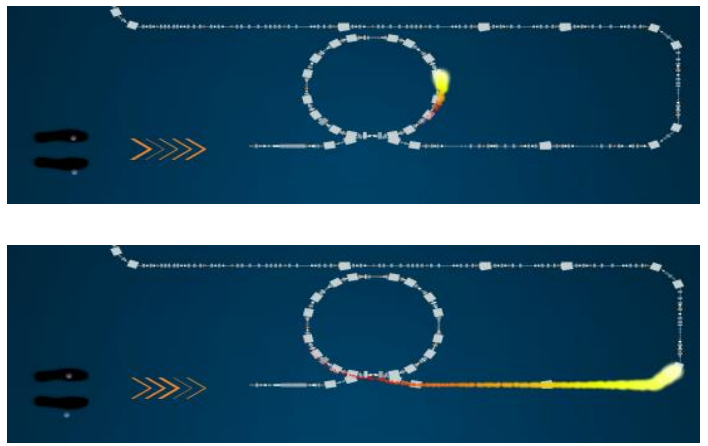

Figure 4c. The "Floor" interface as soon as the user had kicked. The hadron beam gets accelerated.

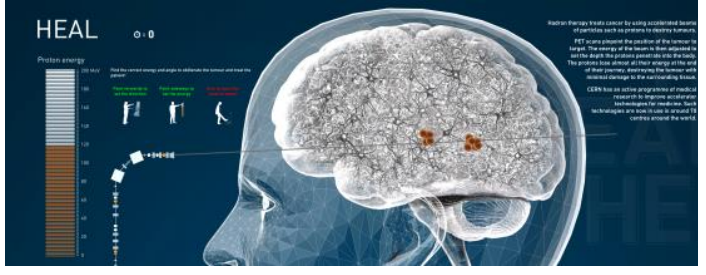

Figure 4b. The "Wall" interface when a player has already selected the energy amount and the direction for the hadron beam to be emitted (green instructions above the corresponding images).

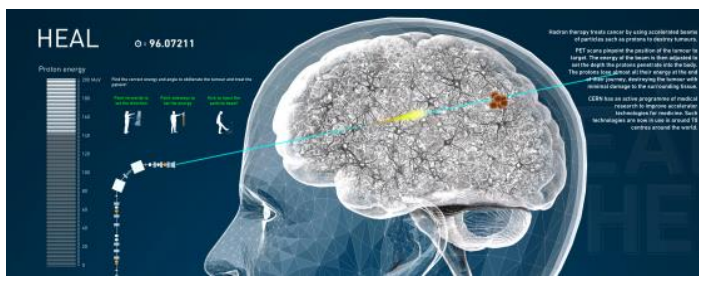

Figure 4d. The "Wall" interface after the kick has happened. The hadron beam is directed to the direction the player has selected before.

Figure 4. HEAL's interface when a gameplay scenario is running

While Proton Football needs external guidance, HEAL can stand by itself as (a) a small info-box is always present to the users providing some main information for Hadron therapy, (b) optical guidance (images) on how to interact with the game controllers is always present as well and (c) a newer version of sensors was used minimising the problems stemming from clothing misinterpretations. 


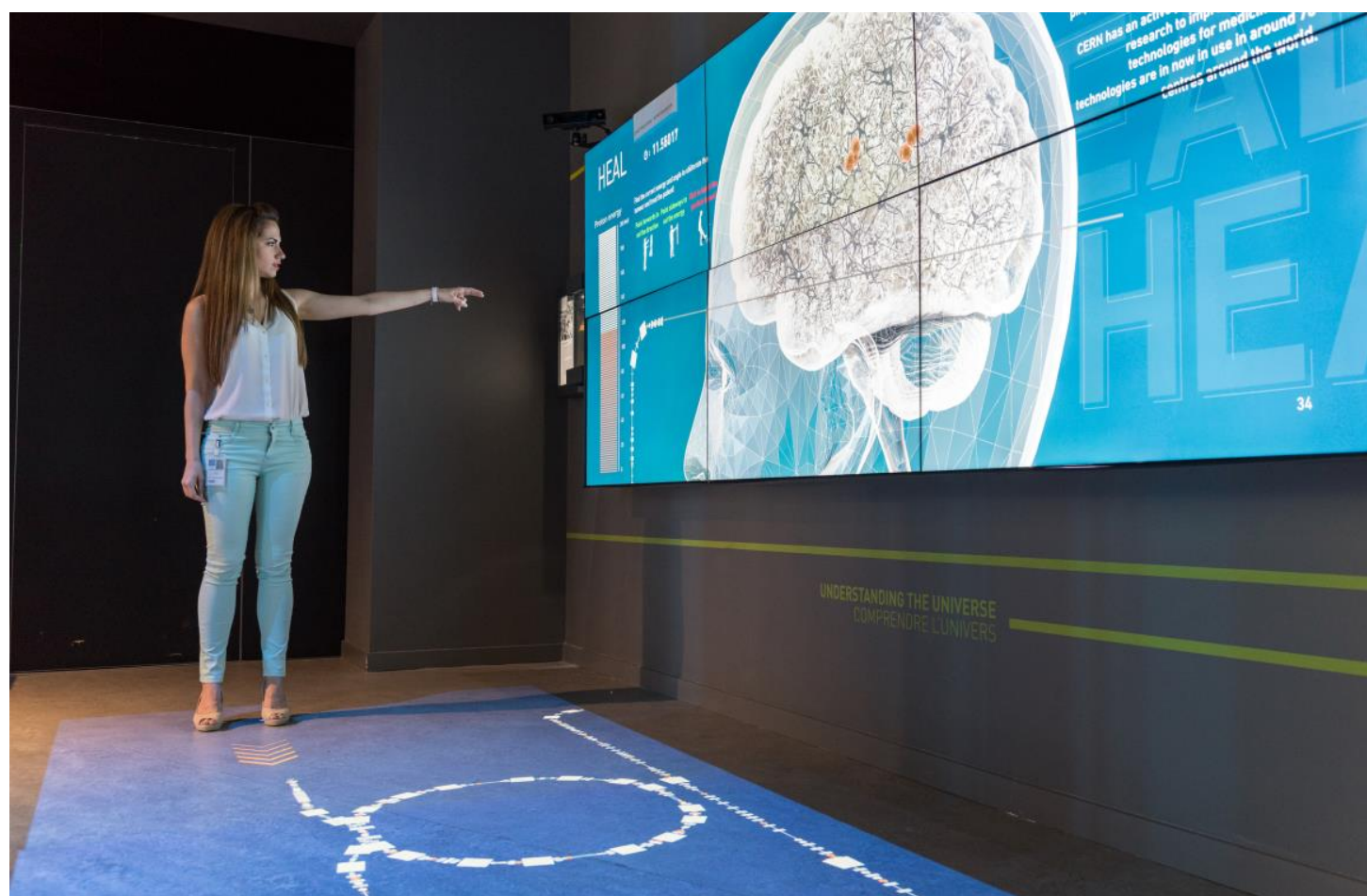

Figure 5. HEAL in Microcosm. User is adjusting the energy to which the hadron beam will be emitted to the humanoid's brain.

As already mentioned above, HEAL has one important difference to the Proton Football game. While to achieve the game goal of Proton Football users need to perform only one single task, kick of a virtual proton and movement of the user's body respectively, in HEAL they need to perform a set of tasks and decide precisely the values needed to set. For example, in Proton Football players need to collide protons with as much energy as they wish. The collision will happen as far as the direction of both protons is correct, regardless of the energy given. However, in HEAL, unless the correct amount of energy and direction is selected, the cancer cells will remain intact.

In addition to the above, Proton Football is a two-player game meaning that the players need to coordinate to achieve a collision. Especially in the case when players want to achieve the best outcome (maximum energy on both protons), both players have to kick with force and at the same line of direction. This means that not only players need to find a playmate, but also manage to coordinate. HEAL is a one-player game as (a) we believe that it diminishes such cases and (b) its representation gets closer to how Hadron Therapy works in real life as hadrons are emitted from one side and not from multiple.

\subsubsection{Questionnaire development}

Questionnaires were developed from findings of previous studies [30-34]. General questions providing basic information on the participants' profiles were included in all questionnaires. In order to cover as many factors affecting the quality of the visitor experience as possible we included the following factors: (a) satisfaction, (b) engagement, (c) learnability, (d) perception, (e) motivation/triggering, (f) ease of use, (g) ease of learning, and (h) gameplay clarity. Since the size of one questionnaire would render it exhausting, we preferred to create a set of questionnaires covering different factors, in order not to discourage a visitor from participating to this preliminary evaluation.

Statements were worded carefully and long, ambiguous, leading and biased questions were avoided. Most of the statements were presented in scales as Likert items from 1 meaning "strongly disagree" to 5 meaning "strongly agree". The questionnaires included demographics questions, as well as open questions that promoted participants to express their opinions on the game.

Questionnaires were available in five different languages: English, French, German, Italian and Greek to increase the number of participants willing to participate. The latest 
pag. 12

evaluation [35] of CERN showed that most of its visitors were speaking one of these languages. By providing questionnaires in the visitors' mother language, misinterpretations would be minimised and visitors would feel more comfortable responding.

Questionnaires were available at a stand next to the HEAL game. Any visitor who has played the game could voluntary fill in a questionnaire. Completed questionnaires were asked to be put in a letterbox next to this stand. This led to a random sample, and not in a sample that was weighted representative of the MIO's visitors. On the other hand, this made the fulfilment of the questionnaire completely voluntary.

In every evaluation there were missing data occurring for different reasons. In this study, we excluded the questionnaires where the respondent has not responded to one or more questions, apart from the two open ones, as well as the ones where the response was invalid (e.g. where only one answer was required but the respondent selected several responses).

Prior to completing the questionnaire, visitor consent was asked. Especially for participants age 16 to 18 , consent from the accompanying adults was required (e.g. parents, teachers, etc.). All questionnaires were anonymous.

\subsubsection{Preliminary evaluation}

A preliminary evaluation of HEAL's prototype was conducted in order to provide us with an assessment of the quality of the user experience. This was necessary to not only evaluate the usability of the game in terms of its user interface and stability, but also to gather data and experience for designing and running a more formal study.

The preliminary evaluation was conducted at Microcosm, CERN's most popular and visited permanent exhibition. HEAL was available in the "Discoveries" area of Microcosm for approximately a month (from March 15th to April 20th 2016) from 8:30 to 17:30. During this period, 221 random visitors in total agreed with a written consent on participating in the evaluation, 142 of who were male $(64.25 \%)$. All data was collected anonymously.

Most of the participants were from 16 to 24 years old (Figure $6-67.87 \%$ ). Organised visits, either school ones or not, were also the most common type of visit (Figure 7 $61.54 \%$ ). All participants were random visitors of Microcosm who could speak fluently and understand the English language.

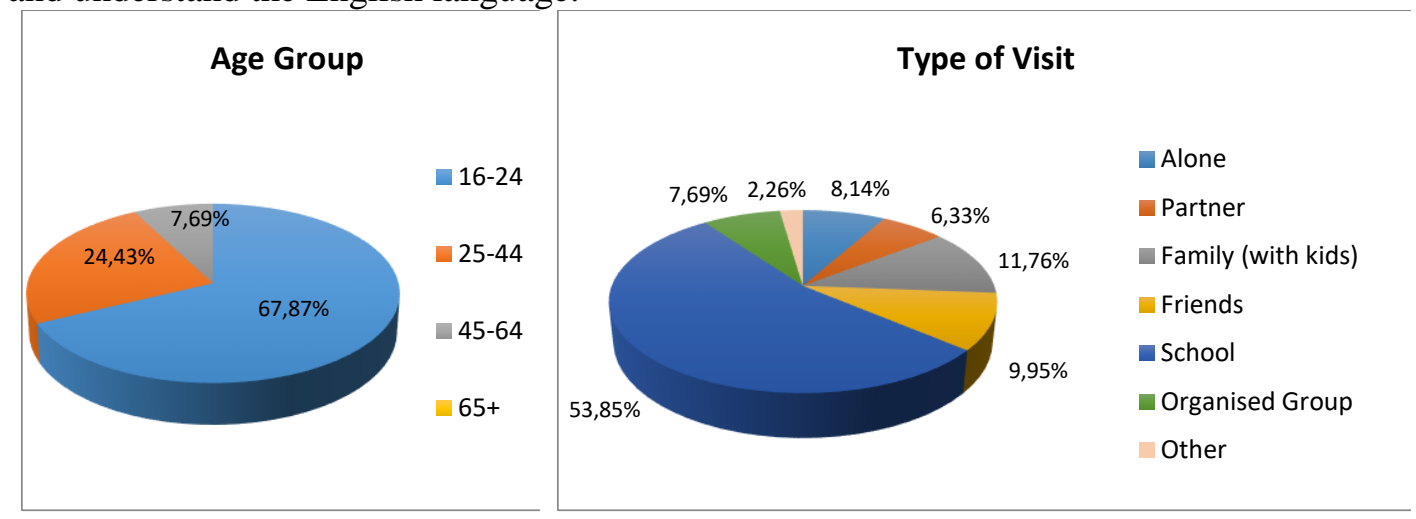

Figure 6. Age group statistics related to the preliminary evaluation.

Figure 7. Type of visit statistics related to the preliminary evaluation.

The first questionnaire included questions for five factors:

- Satisfaction: whether the game is interesting to use and met the expectations of its users

- Engagement: whether the game was engaging enough to keep playing

- Learnability: whether the information is presented in meaningful ways

- Qualia: whether the game provided a conscious experience

- Motivation/Triggering: whether the game triggered its users to learn more about its topic after their visit. 
The second questionnaire included questions for four factors:

- Ease of use: how quickly expert users can accomplish tasks once they have learned to play the game

- Ease of learning: how easy it is for first time novice and casual users to figure out how to play the game

- Gameplay Clarity: whether the game contained all information necessary to evaluate a position and determine the correct play

- Overall Satisfaction: the user's overall satisfaction with the game experience.

Since both questionnaires comprised of a big number of questions, we will only present the ones with the clearest results or the ones that affected the HEAL's architecture before implementing a more formal evaluation.

The results of the preliminary evaluation were encouraging as HEAL seemed to be very appealing to everyone, especially teenagers. The prototype provided a fun game environment while providing enough information for users to understand (a) the purpose of the game and (b) how it is connected to CERN, and discern (c) the problem presented and (d) the solution offered, thus leaving CERN (e) having an overview of the entire method of treatment.

Our observations identified the interactivity provided as a particularly attractive design feature. Visitors often became more excited as they realised they could interact with the game controls just by using body movements $(85.45 \%)$. This could be one of the reasons participants described the game as fun $(99.09 \%)$ and interesting $(94.55 \%)$, while being educational by introducing some concepts about the angle and the amount of energy needed to succeed in the game. In total, 158 participants shared their thoughts on what they liked about HEAL. Table 1 shows the most frequent comments.

Table 1. Participants' comments in open question related to the strong points of the HEAL game.

\begin{tabular}{c|c|c|c}
\hline What visitors liked & $\#(\%)$ & What visitors liked & $\#(\%)$ \\
\hline Interactivity & $55(34.81)$ & Simplicity & $7(4.43)$ \\
\hline Fun / Enjoyable & $24(15.19)$ & Clarity & $6(3.80)$ \\
\hline Graphics & $15(9.49)$ & Ease of Learning & $6(3.80)$ \\
\hline Educational / Informative & $11(6.96)$ & Easy to play & $5(3.16)$ \\
\hline Understandable & $10(6.33)$ & Usability & $5(3.16)$ \\
\hline Gameplay & $10(6.33)$ & Connection with medicine & $4(2.53)$ \\
\hline Interesting & $9(5.70)$ & Fun to learn & $3(1.90)$ \\
\hline
\end{tabular}

Participants have found HEAL easy and simple to use (92.79\%). Regarding the ease of learning, statistics were really encouraging. Most of the participants stated that HEAL was easy to learn to play $(97,30 \%)$ and didn't think that a lot of things were essential to learn before they could play the game $(89,49 \%)$.

It is important to also mention that only $36,94 \%$ of the participants admitted having a strong experience with the X-Box Kinect or Wii [36] sensors. Since more than half of the participants $(63,06 \%)$ had no strong experience with either the X-Box Kinect or Wii sensors, it is quite interesting to take a deeper look into their game experience regarding the ease of learning. Most of the participants $(98,11 \%)$ stated that HEAL was easy and fast to learn to play. In addition, participants admitted becoming quickly good at it $(84,91 \%)$ and mentioned that the controls of the game were not difficult to understand $(92,45 \%)$. In other words, regardless of the users' previous experience with either the XBox Kinect or any other similar sensors, the preliminary evaluation results show that HEAL fulfilled its purpose regarding the ease of learning.

As mentioned above, HEAL is a single player game. When asked, more than half of the participants (53.64\%) agreed that a two-player competitive version of HEAL would be more entertaining (Figure 8). However, most of them $(82.81 \%$ ) admitted that the single 
pag. 14

player version is more suitable since it describes better how hadron therapy works in real life. As shown in Figure 9 more than half of the total participants (58.18\%) noted the single player version as the best describing one.

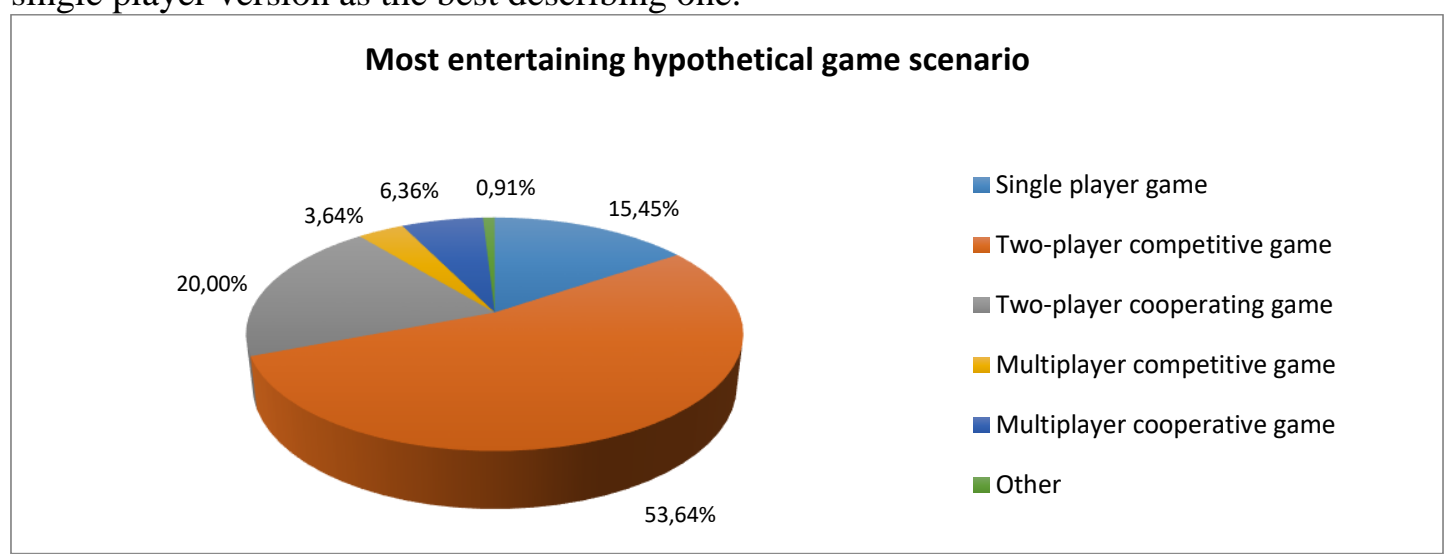

Figure 8. Participants' answers related to the most entertaining hypothetical game scenario.

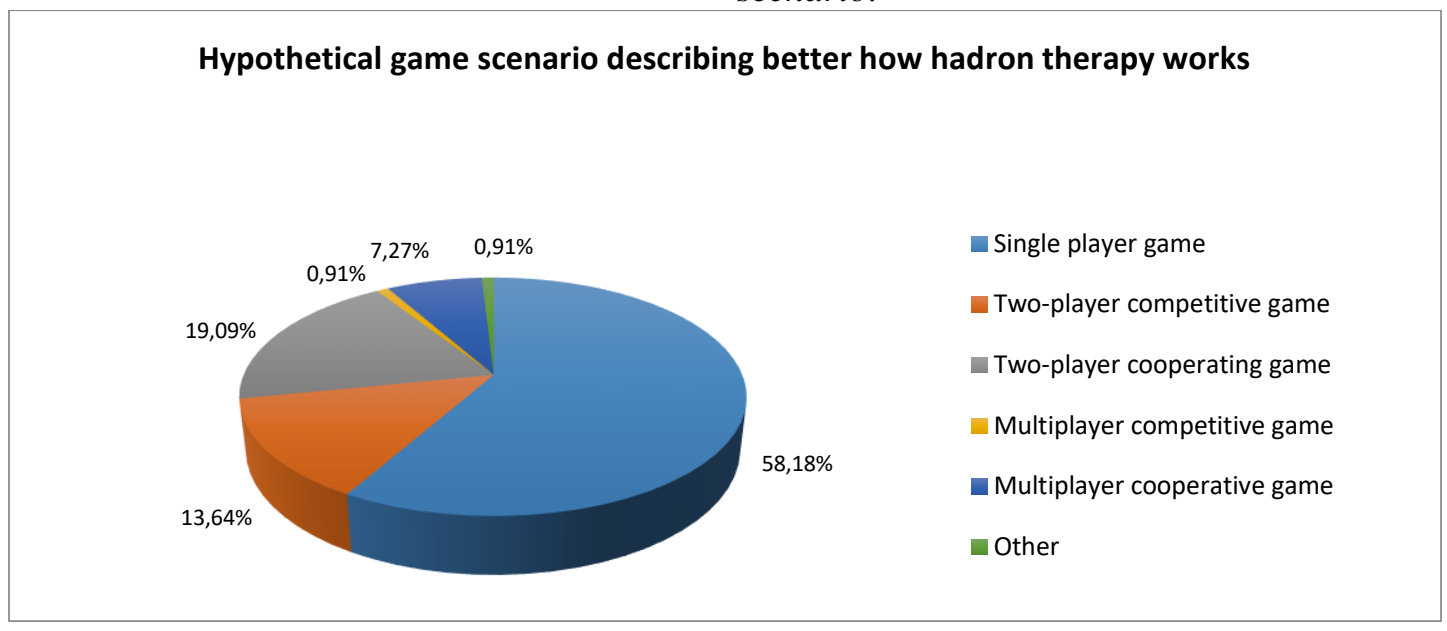

Figure 9. Participants' answers related to the best describing hypothetical game scenario.

The preliminary evaluation also revealed some minor bugs in the game prototype that were later eliminated and tested before the formal evaluation of HEAL. The bugs were mainly related to the way the game was recognising players and their movements, especially when another visitor was entering the interactive space. In total, 103 participants shared their thoughts on how HEAL should be improved. Table 1 shows the most occurred suggestions for improvement by users. On the top of the list, users expressed the need of further explanation of what HEAL is about and better instructions on how to play the game. To achieve that, a more detailed description of Hadron Therapy, as well as images accompanying the existing text instructions on how to play the game were added to the game interface. Problems related to the sensors were identified and eliminated. Minor changes have also been applied to the gameplay since some participants seemed to find the idea of representing cancer growing over time unpleasant. Finally, suggestions such as climaxing difficulty were not adopted as we believe that a game such as HEAL should be fast to play and simple enough to not keep the users more than two minutes as explained before. 
Table 2. Participants' comments in open question related to the points that need improve in the HEAL game.

\begin{tabular}{c|c|c|c}
\hline Suggestion for Improvement & $\#(\%)$ & Suggestion for Improvement & $\#(\%)$ \\
\hline More instructions / explanation & $28(27.18)$ & Multiplayer & $6(5.83)$ \\
\hline Improve sensor accuracy / sensibility & $22(21.36)$ & $\begin{array}{c}\text { Difficulty } \\
\text { (Harder to win) }\end{array}$ & $6(5.83)$ \\
\hline Improve Kinect reception & $13(12.62)$ & Improve aiming precision & $6(5.83)$ \\
\hline Better input feedback & $6(5.83)$ & $\begin{array}{c}\text { Climax difficulty } \\
\text { (More levels) }\end{array}$ & $3(2.91)$ \\
\hline
\end{tabular}

After playing HEAL, more than half of the participants expressed their desire to learn more about science $(61.82 \%)$, or CERN technologies $(79.09 \%)$, or specifically hadron therapy $(76.36 \%)$. Ten out of fourteen people stated that after playing HEAL their stance was changed. While before they were not interested in CERN, after playing HEAL, ten (71.42\%) claimed wanting to learn more about hadron therapy and eleven $(78.57 \%)$ about science and CERN technologies.

In addition to the above, many where the participants that would like to play longer $(60.91 \%)$ or again $(82.73 \%)$. However, results have shown that visitors usually do not come back for a second visit. Many were the cases when people would come from a different country, or even a different content, in order to visit the exhibition. Out of 221 participants, only 5 admitted having visited the organisation before. For the rest of the participants (97.74\%) this was the first time visiting CERN. With such a high percentage of first-comers, CERN needs to make sure that whatever message they would like to pass to its visitors, it should happen during their visit time. This also means that visitors would neither have played the game before, nor play with it ever again, or at least in the near future. On the other hand, participants were willing to recommend HEAL to a friend $(82.27 \%)$, but only some of them $(21.82 \%)$ would like to share their score (time it took someone to vanish all cancer cells) on Social Media.

Overall, almost all responders stated having a good experience (Good $-43.89 \%$, Excellent $-53.85 \%$ ) playing HEAL.

\subsubsection{Formal Evaluation}

The formal evaluation of HEAL took place one week after the preliminary evaluation. Using the latter one's data and experience, the formal study was designed to cover any missing spots or clarify uncertainties the preliminary evaluation showed. We have also used selected questions from previously validated questionnaires found in [37]. In addition, we performed a Cronbach's alpha analysis to test the internal consistency of the questionnaire we used. The value found was 0.62 which is a good, acceptable value for internal consistency.

Questionnaires [38] were available at a stand next to the HEAL game. Any visitor who has played the game could voluntary fill in a questionnaire. Completed questionnaires were asked to be put in a letterbox next to this stand. Similarly to the preliminary evaluation, the fulfilment of the questionnaire was completely voluntary.

The questionnaires available to the participants were much shorter but included more targeted and essential questions, focusing on evaluating the user experience and the learning processes used. Statements were again worded carefully and long, ambiguous, leading and biased questions were avoided. For almost all questions participants had to select one of the possible answers. There was also one open question that asked participants to express their life experience, allowing respondents to formulate their own statements. In this study, we excluded the questionnaires where the visitor has not responded to one or more questions, apart from the open question, as well as the ones where the response was invalid (e.g. where only one answer was required but the respondent selected several responses).

The formal evaluation was conducted at the same place as the preliminary evaluation, CERN's permanent exhibition called Microcosm, and it was conducted over a two-month period (from April 28th to July 1st 2016). During this period, 463 random visitors agreed 
pag. 4

with a written consent on participating in the formal evaluation, 259 of who were male $(55,94 \%)$. Figure 10 shows that most of the participants were from 16 to 24 years old $(54,86 \%)$. All participants were random visitors of Microcosm who could speak fluently and understand the English language. All data was collected anonymously.

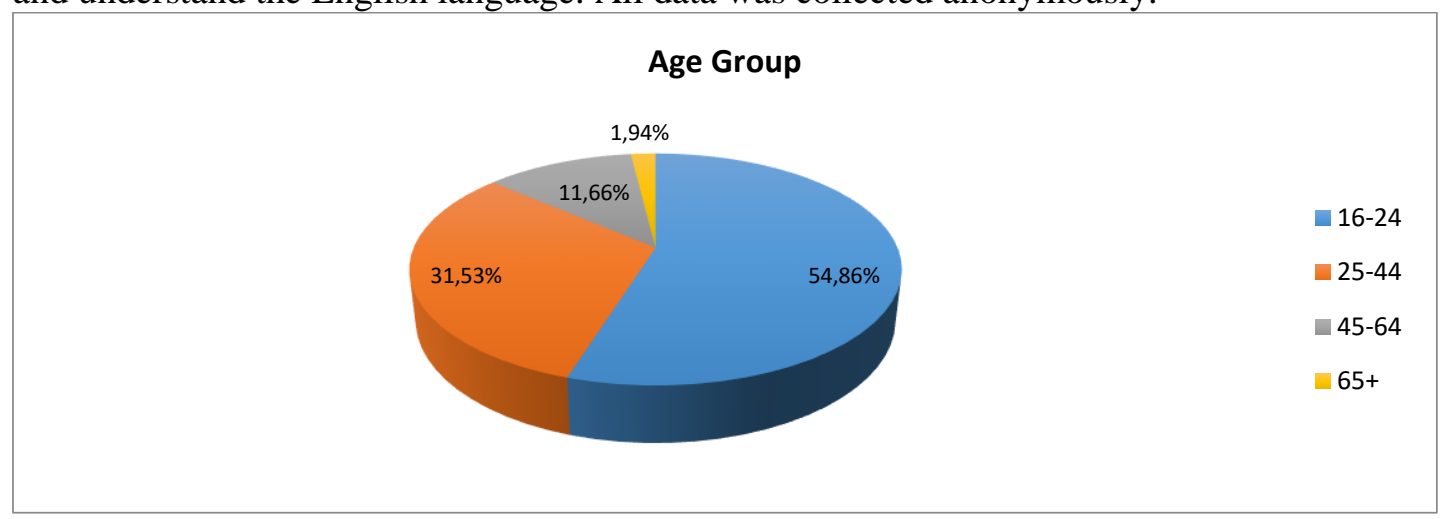

Figure 10. Age groups of the participants who took part in the formal evaluation

In order to evaluate the quantity and quality of information assimilated by participants, a couple of questions related to the background knowledge they had before visiting Microcosm, were added to the questionnaire. Results related to learnability were promising.

As shown in Figure 11 and Figure 12 while a quite more than a half $(67.17 \%)$ participants knew about CERN's contribution to society (e.g. WWW, Grid), only $38.88 \%$ of them had heard about proton therapy. As a matter of fact, only three out of ten people knew both about CERN's contribution and Proton Therapy before their visit $\left(\chi^{2}(1)=18.338, \mathrm{p}=0\right)$ at Microcosm while almost one fourth of the participants knew nothing about CERN's activities outside the physics field. Also, male visitors seem to know more about CERN's contribution to society before their visit $\left(\chi^{2}(1)=19.282, p=0\right)$. These results prove the urgent need of informing the public about the organisation's contribution to society besides physics.

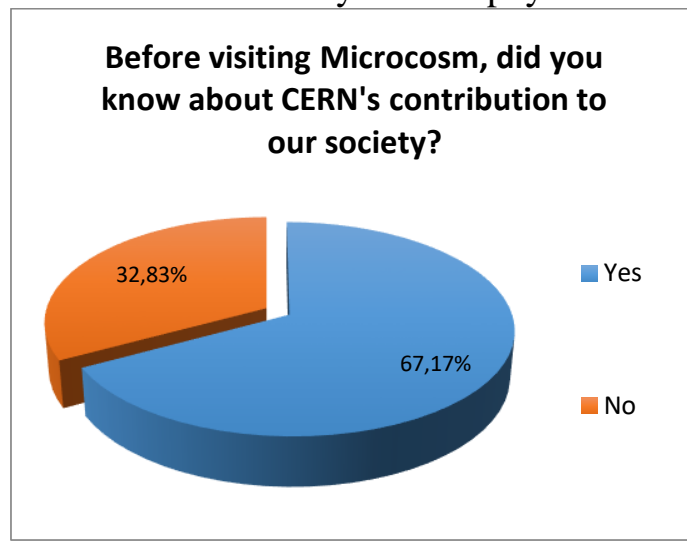

Figure 11. Visitors background knowledge on CERN's contribution.

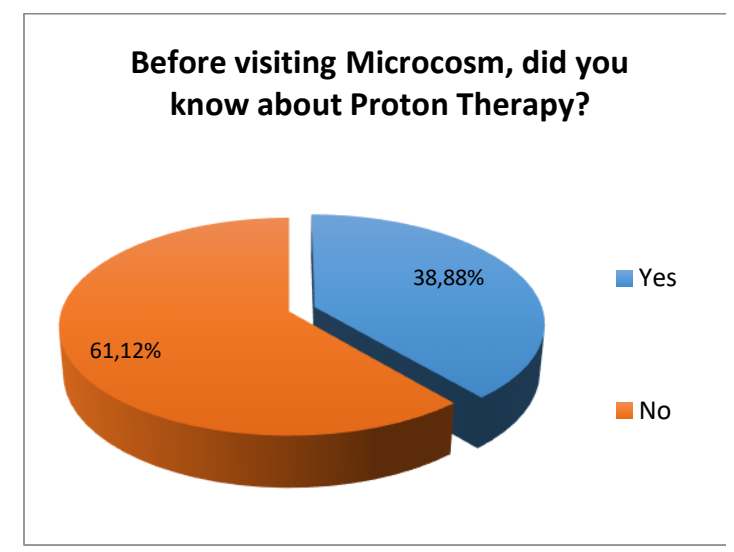

Figure 12.Visitors background knowledge on Proton Therapy.

After interacting with HEAL, participants were asked about the information acknowledged by using the nano-game (Figure 13). Almost $80 \%$ of the participants claimed understanding what Hadron Therapy is and how it works by using HEAL. Only $3.67 \%$ of the participants said that the presentation of Hadron Therapy was not clear. Moreover, most participants (92.22\%) suggested the more frequent use of such games in learning. 


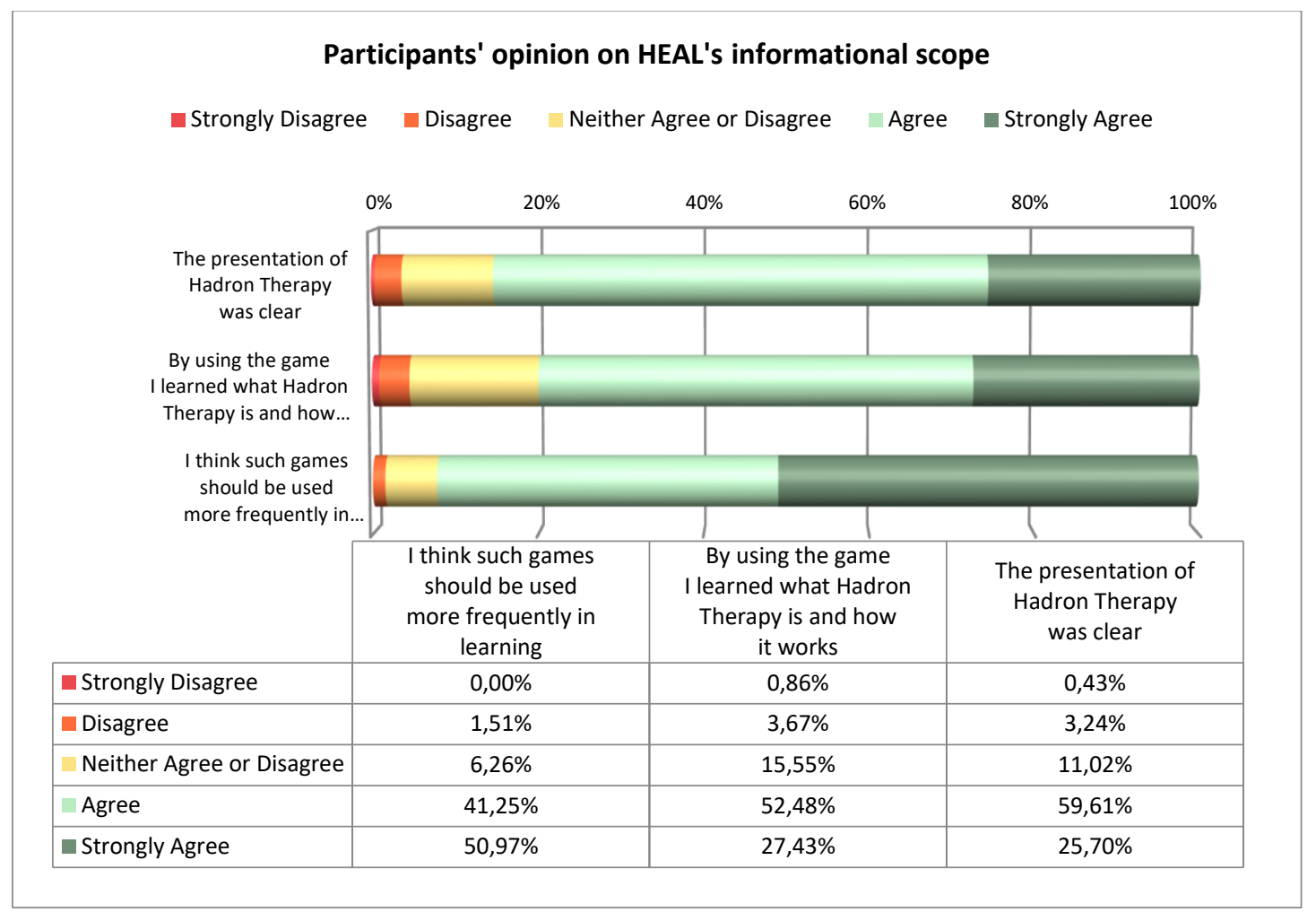

Figure 13. Participants' opinion on HEAL's informational scope.

To examine whether HEAL fulfilled its informational purpose and participants actually have drawn the information intended to transmit through the nano-game, it was essential to evaluate whether participants expressed their honest opinion. In an effort to achieve that and eliminate random answering, we included three questions related to hadron therapy and how it works, as well as justification questions asking to select an explanation for participants' answers in the main questions.

The questions were chosen carefully to reflect the main messages HEAL aims to pass to its users. The first and second questions focused more on the way Hadron Therapy works and its superiority to conventional therapy, while the third question was related to CERN's contribution to society. The first two questions were followed by justification questions, asking the participants to state the elements of the game that shaped their opinion.

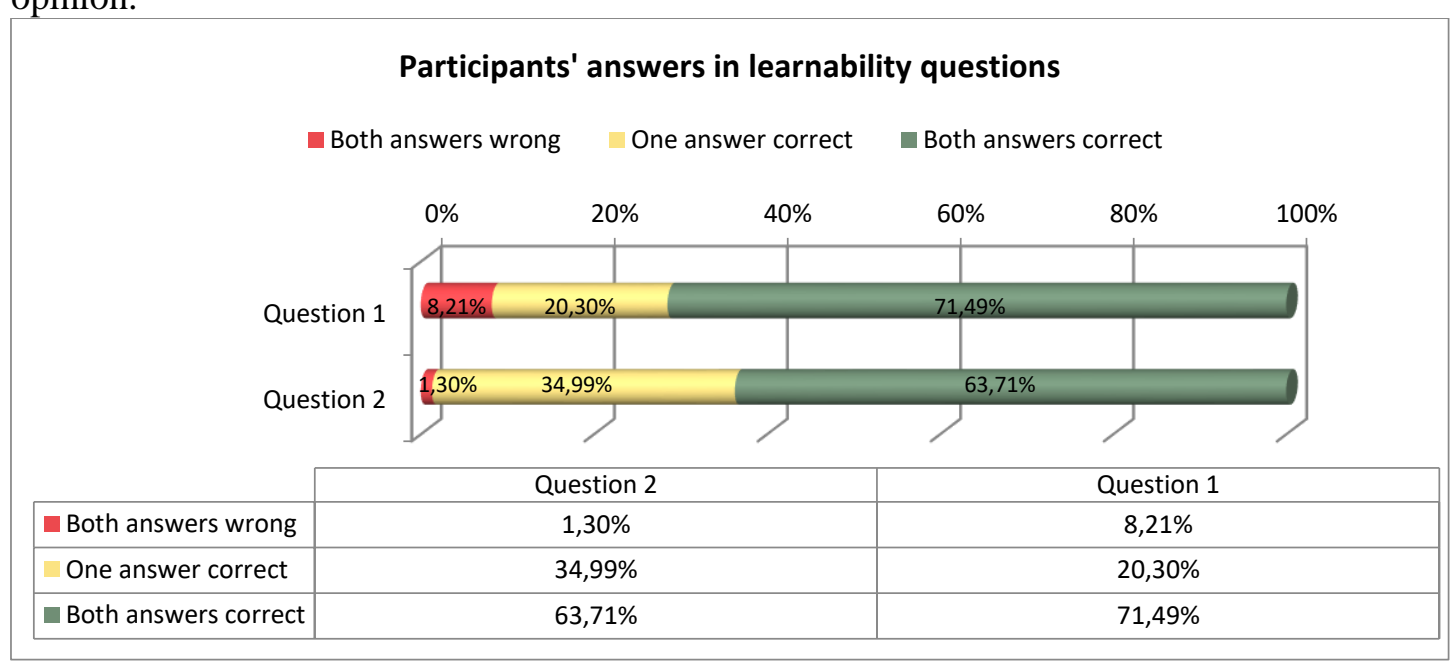

Figure 14. Participants' answers in the first two learnability evaluation questions.

From participants' answers it seems that the majority of participants apprehended the information that HEAL provided them. This could be either from its gameplay metaphors 
pag. 6

(e.g. aiming, kicking, etc.), or from its graphical interface. Almost two thirds (71.49\%) of the participants answered correctly in both the first question and its justification, while more than half $(63.71 \%)$ in both the second question and its justification. Finally, almost all participants $(97.41 \%)$ answered correctly in the third question. It should also be mentioned that male participants responded correctly to the first question (and its justification) $\left(\chi^{2}(1)=5.052, \mathrm{p}<0.05\right)$. Participants who claimed knowing about CERN's contribution before their visit have also responded correctly to the first question (and its justification) $\left(\chi^{2}(1)=6.539, \mathrm{p}<0.05\right)$.

In case a participant answered a question correctly but the justification of this exact question wrongly, or in reverse, we considered the answer to that question as wrong. If a participant would grasp the deep meaning of the concepts, she would have been able to reply correctly to both questions. Figure 14 shows participants' answers regarding the first two questions, which were followed by verification questions. The green colour represents the participants that replied correctly both to the question and its justification question. The yellow colour represents the participants that answered only one of the questions correctly (either the main or the justification question). Finally, the red colour represents the participants who answered both the main and the justification question wrongly. It is quite evident that the number of participants having both the question and its verification wrongly answered is really small.

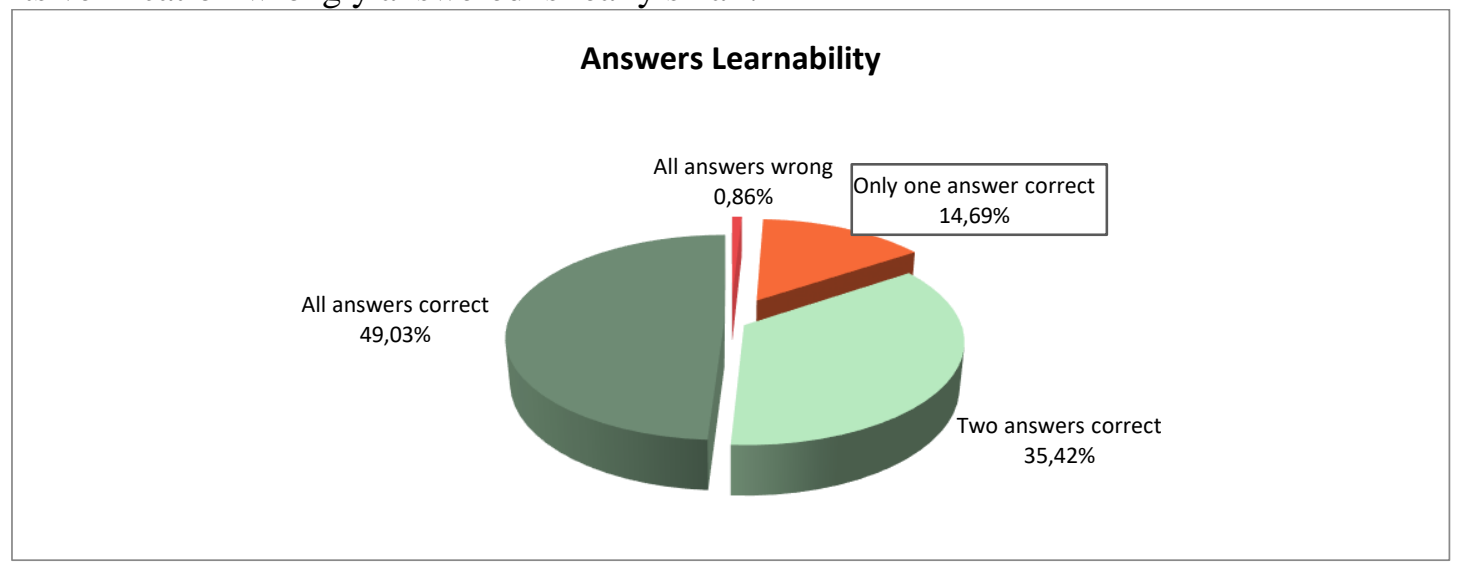

Figure 15. Statistics related to users' responses in evaluating the learning outcome.

Having the above-mentioned assumption in mind, only four participants answered all three questions wrong $(0.86 \%)$, while almost half of the participants answered all questions correctly (49.03\%). Figure 15 shows the number of the total correct answers participants gave.

Consequently, as shown by participants' answers, HEAL did fulfil its learning purpose since participants claimed of having learnt what Hadron Therapy is and how it works. This also gets verified from their correct answering in a set of learnability questions $\left(\chi^{2}(12)=44.547, \mathrm{p}=0\right)$. The evaluation showed that only $11.66 \%$ of participants had a wrong perception of Hadron Therapy. This is because although they claimed having a good understanding of what Hadron Therapy is and how it works after playing the game, they replied to more than half of the questions wrongly.

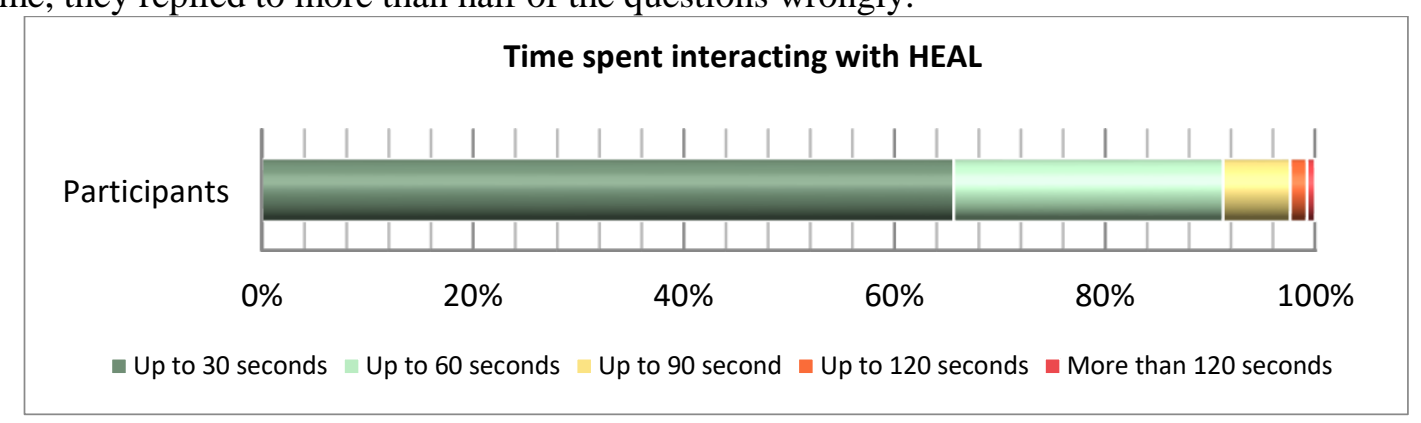

Figure 16. Time participants spend playing HEAL. 
It is also worth mentioning that for the first 125 random participants the time spent interacting with HEAL was also recorded by the evaluator. Figure 16 shows how much time participants spent using the game. It is quite impressive to mention that fifty two out of one hundred and twenty five participants answered correctly to all learnability questions by only spending no more than 30 seconds of interaction with HEAL (41.60\%). This number gets much higher for participants who spent up to 60 seconds $(77 / 125$ $61.60 \%$ ). Table 3 shows the number of correct answers given relatively to the time spend interacting with HEAL. While the sample may be not be big enough, there is a distinct trend that most users acquire the needed knowledge during their first minute of interaction with HEAL. A couple of tries with the game seem to be enough in order for participants to understand what Hadron Therapy is and how it works. This seems to be the same case even when users get the wrong perception of a game's purpose. Playing longer HEAL did not lead into users getting a clearer understanding. On the contrary, users' perception was formed during the first minute of interaction, similarly to the first case. This means that users acquire their perception during their first minute of interaction with the game, regardless of whether this is correct or not.

Overall, $92.01 \%$ of the participants stated having a good experience (Good $-63.07 \%$, Excellent - 28.94\%) playing HEAL (Figure 17).

Table 3. Number of correct answers related to time spent playing the game for the first 125 random participants.

\begin{tabular}{c|c|c|r|c}
\hline Time in Seconds & All three correct & Two correct & One correct & None correct \\
\hline Up to 30 & $52(41,60 \%)$ & $21(16,80 \%)$ & $8(6,40 \%)$ & $1(0,80 \%)$ \\
\hline Up to 60 & 仓 $77(61,60 \%)$ & 仓 $28(22,40 \%)$ & $8(6,40 \%)$ & $1(0,80 \%)$ \\
\hline Up to 90 & 仓 $83(66,40 \%)$ & 仓 $29(23,20 \%)$ & 仓 $9(7,20 \%)$ & $1(0,80 \%)$ \\
\hline Up to 120 & 仓 $85(68,00 \%)$ & $29(23,20 \%)$ & $9(7,20 \%)$ & $1(0,80 \%)$ \\
\hline More than 120 & $1(0,80 \%)$ & $0(0,00 \%)$ & $0(0,00 \%)$ & $0(0,00 \%)$ \\
\hline
\end{tabular}

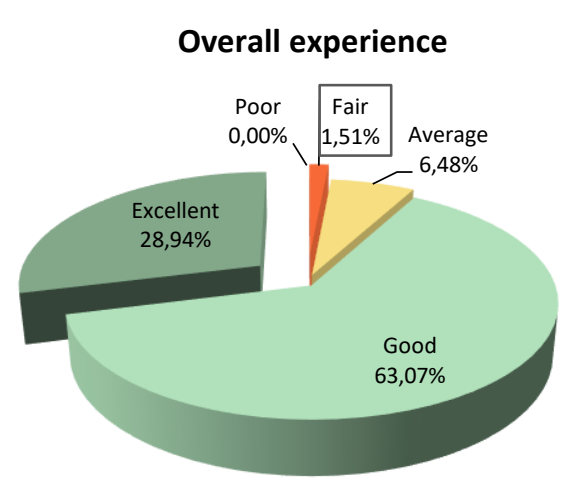

Figure 17. Statistics related to the overall experience of the users during the formal evaluation.

\section{Discussion}

HEAL's evaluation showed some promising results when put under the pressure of loads of visitors. Microcosm has 400 visitors in average per day and its open for 9 hours on working days, and for 8 hours on Saturdays. This means that the MIO needs to be able to pass its message to as many visitors as possible and as fast as possible. This gets more challenging when visitors are part of group visits. More than 5 people (even 20) can arrive at the same time and place and, since a visit lasts approximately 1.5 hours, they only spend a few minutes at one exhibit. Since the majority of the visitors have never visited 
pag. 8

CERN before and one fourth of its visitors has no knowledge about CERN's activities outside the physics field, the challenge to deliver the main message gets even more elusive. This can be feasible with nano-games. Doing some simple calculations, on a working day having 400 visitors, each one can play HEAL spending 1 minute and 21 seconds. Having in mind the evaluations' results, 1:20 minutes is more than enough for a visitor to get the main message that the nano-game is willing to pass to its players. This is verified by the fact that participants who agreed that the hadron therapy presentation was clear also responded correct to all learnability questions $\left(\chi^{2}(12)=44.547, p=0\right)$.

In addition to the above, a MIO can have more than one nano-games. Since its purpose is fulfilled in a matter of seconds, visitors can play a number of nano-games and acquire in a playful way the needed information they were seeking for. This would not be possible with games that last tens of minutes or even need training in order to be played. As already presented in [22], micro-games are described as a "means to reinforce and integrate part of the knowledge learnt in the expository lecture" and are lectures' supplements. For their evaluation, students from four high schools participated answering a) an immediate post-questionnaire, and b) a one month delayed post-questionnaire. The full questionnaires were not available in [22]. Results seemed encouraging as the microgame was at least as effective as traditional instruction, when measured immediately after the exposure and reinforced and integrated knowledge was retained better. On the other hand however, nano-games are presented as an independent, self-contained approach, with no need for introduction or briefing, either before of after their use. While an infobox was available, it does not imply that users actually took the time to read it, as no data were collected to this end. In addition to the above, our evaluation took place in an international organisation, CERN, visited by a high variety of visitors in the terms of several aspects such as sex, gender, nationality, background knowledge, etc. The formal evaluation showed that 350 out of 463 (75.59\%) participants come from countries outside Switzerland, France, Germany, Italy and Austria to visit CERN. As a result, one can assume that due to the long-distance limitation, visitors are not able to revisit often. This, along with the anonymity of the questionnaires, makes the possibility of postquestionnaires for our evaluation unattainable.

Another aspect that had not been mentioned above could be the cost of developing a nano-game. Nano-games are self-contained games of a single level of difficulty, contrary to other games where more than one difficulty levels are available. Usually, small MIOs do not have the economic vigour (e.g. human resources) to fund expensive games. Nanogames offer the alternative of an inexpensive, yet qualitative implementation since due to their shortness and simplicity, one does not need to develop as much as they would when developing a multilevel game.

Finally, during our evaluation there were a few cases where visitors had physical disabilities, either temporary (broken arm or leg) or permanent (paralysed hand or leg). In case one of their hands was functional, visitors were able to play the game with no issues. There were also cases where visitors would use a wheelchair. HEAL was able to identify the user and, albeit the user was not able to physically kick, just by moving the wheelchair forward they were able to simulate the "kick" gesture and be able to play. Even though HEAL responded well in such cases and is optimistic, we believe that the cases we had are not enough to draw conclusions, so we leave it as future work.

\subsection{Challenges and guidelines}

The main challenge of developing a nano-game is identifying the message and selecting a suitable metaphor to help pass the message across. To this end, a team of experts on the subject and designers is of the essence. First, experts have to come down to a main message that can be written in a single phrase. A first guideline is to start designing only when this message is identified and agreed upon. In the case of HEAL game, the phrase to pass was "It takes careful planning to hit a tumour target". In the case of Proton football the phrase was "It is very difficult to make two protons collide". Other, secondary messages can be written down to see if the selected metaphor can cater for them, without 
obscuring the main message. Secondary messages for HEAL game were: "The new therapy can be very efficient" and "A particle accelerator can be used for treatment".

The second major challenge is to identify a suitable metaphor. For our nano-games a full body gesture-based environment is being used. The basic guideline calls for metaphors based on universally understood and accepted gestures. In the case of Proton football, the metaphor was the kicking of a ball (hence the title of the game). In the case of HEAL game, the metaphor was initially that of an archer. But, technical difficulties changed the metaphor to that of "point to an object". Which reveals the next challenge: the metaphor has to be programmatically implemented and technology has its limits.

Another major challenge is not to convey dubious or wrong messages. For example, while considering whether HEAL game should be a single or two-player game and having implemented the popular Proton football game, the first notion was to re-use the football metaphor. But, that would probably mislead players into thinking that the tumor was being attacked from multiple sources. Finally, to avoid issues derived from the two-player game, as previously described, it was decided to adopt a single player type of game. However, in case of a two-player configuration, the tasks would have to be redistributed to allow two players to play. Again, the guideline calls for early evaluation of the designed prototype, even as a mock up, to avoid unwanted messages. One has to remember that the selection of graphics can greatly help in that. Simple, intuitive graphics have to be selected and unnecessary clutter has to be avoided. A new user, who has to learn the game in tens of seconds, has to get information for the task she has to perform, supported in focusing on it. She should not get distracted with additional information.

\section{Limitations - Future Work}

To conclude, we believe that nano-games offer MIOs the opportunity to provide a fast, yet qualitative informational experience to their visitors. Regardless of their shortness in time or of the crowds that may exist, especially in peak hours, visitors are able to get the maximum interactive experience possible in a matter of seconds. Moreover, MIOs ensure that every visitor leaves their venue with an understanding of what they wished to communicate through a fun medium. For a MIO like CERN, where the exhibitions have a strong basis on physics, full-body interactive nano-games can offer a viable approach to help convey the message to the visitors.

However, one could envisage the usage of nano-games in the context of other types of MIOs, in fields other than physics. For example, nano-games could be exploited in history of sports museums to offer insights on games mechanics or ergonomics. Another example could be in a geology museum to help understand the pressure and heat required to create a diamond.

Working in a real life setting in a crowded venue like CERN, we had to respect organisational issues and not interrupt the visitor flow. For this reason, we proceeded with a grab sampling process. This type of sampling process, being non-probability, implies that it is more flexible in a real venue setting, but it also means that we cannot easily generalise the findings. Keeping these in mind, the nevertheless strongly significant results of the present work, can be read as strong indicators of possible tendencies. However, the present work also used a rather large sample $(\mathrm{N}=463)$ to increase the reliability of its findings.

In the near future we are planning to test the suitability of nano-games to other more traditional MIOs, especially museums and cultural venues. We foresee that, depending on the MIO, special effort may be required to identify the main message to be conveyed through the nano-game and design nano-games that manage to communicate the message to the visitors. Also, we are looking into using small demo videos or animations, instead of images, on how to play the nano-games. The demo videos will be automatically 
pag. 10

triggered as soon as a visitor enters the interactive space aiming to attract her to play the game.

\section{References}

[1] F. Masciarelli, "Museums in the digital era: Technology and Innovation," Dipartimento di Economia e Finanza Cattedra di Entrepreneurship, Innovation, and

Technology, 2017.

[2] J. H. Falk and L. D. Dierking, The museum experience revisited: Routledge, 2016.

[3] J. M. Randel, B. A. Morris, C. D. Wetzel, and B. V. Whitehill, "The Effectiveness of Games for Educational Purposes: A Review of Recent Research", Simulation \& Gaming, vol. 23, pp. 261-276, 1992, doi: 10.1177/1046878192233001.

[4] K. E. Ricci, E. Salas, and J. A. Cannon-Bowers, "Do Computer-Based Games Facilitate Knowledge Acquisition and Retention?", Military Psychology, vol. 8, pp. 295-307, 1996, doi: 10.1207/s15327876mp0804_3.

[5] (2018) Samsung Great Court Games. Available: http://www.britishmuseum.org/learning/samsung centre/samsung great court ga mes.aspx

[6] E. Klopfer, J. Perry, K. Squire, M.-F. Jan, and C. Steinkuehler, "Mystery at the museum", pp. 316-320, 2005, doi: 10.3115/1149293.1149334.

[7] Q. Sun, C. Ardito, P. Buono, M. F. Costabile, R. Lanzilotti, T. Pederson, et al., "Experiencing the Past through the Senses: An M-Learning Game at Archaeological Parks", IEEE Multimedia, vol. 15, pp. 76-81, 2008, doi: 10.1109/mmul.2008.87.

[8] B. Serrell, "Paying attention: The duration and allocation of visitors' time in museum exhibitions", Curator: The museum journal, vol. 40, pp. 108-125, 1997, doi: 10.1111/j.2151-6952.1997.tb01292.x.

[9] C. Sandifer, "Time-based behaviors at an interactive science museum: Exploring the differences between weekday/weekend and family/nonfamily visitors", Science Education, vol. 81, pp. 689-701, 1997, doi: 10.1002/(sici)1098237x(199711)81:6<689::aid-sce6>3.0.co;2-e.

[10] J. H. Falk, "Analysis of the Behavior of Family Visitors in Natural History Museums: The National Museum of Natural History", Curator: The Museum Journal, vol. 34, pp. 44-50, 1991, doi: 10.1111/j.2151-6952.1991.tb01454.x.

[11] J. Stevenson, "The long-term impact of interactive exhibits", International Journal of Science Education, vol. 13, pp. 521-531, 1991, doi: 10.1080/0950069910130503.

[12] J. Diamond, "The Behavior of Family Groups in Science Museums", Curator: The Museum Journal, vol. 29, pp. 139-154, 1986, doi: 10.1111/j.21516952.1986.tb01434.x.

[13] P. M. McManus, "It's the company you keep ...: The social determination of learningrelated behaviour in a science museum", International Journal of Museum Management and Curatorship, vol. 6, pp. 263-270, 1987, doi: 10.1080/09647778709515076.

[14] D. L. Boisvert and B. J. Slez, "The relationship between exhibit characteristics and learning-associated behaviors in a science museum discovery space", Science Education, vol. 79, pp. 503-518, 1995, doi: 10.1002/sce.3730790503.

[15] C. A. Cone and K. Kendall, "Space, Time, and Family Interaction: Visitor Behavior at the Science Museum of Minnesota", Curator: The Museum Journal, vol. 21, pp. 245258, 1978, doi: 10.1111/j.2151-6952.1978.tb00545.x.

[16] G. Chiozzi and L. Andreotti, "Behavior vs. Time: Understanding How Visitors Utilize the Milan Natural History Museum", Curator: The Museum Journal, vol. 44, pp. 153165, 2001, doi: 10.1111/j.2151-6952.2001.tb00038.x.

[17] A. Antoniou, I. Lykourentzou, J. Rompa, E. Tobias, G. Lepouras, C. Vassilakis, et al., "User profiling: Towards a Facebook game that reveals cognitive style," in International Conference on Games and Learning Alliance, 2013, pp. 349-353, doi: 10.1007/978-3-319-12157-4_28.

[18] Y. Naudet, I. Lykourentzou, E. Tobias, A. Antoniou, J. Rompa, and G. Lepouras, "Gaming and cognitive profiles for recommendations in museums," presented at the 
8th International Workshop on Semantic and Social Media Adaptation and Personalization (SMAP), 2013.

[19] J. N. Neto, R. Silva, J. P. Neto, J. M. Pereira, and J. Fernandes, "Solis' Curse-A Cultural Heritage game using voice interaction with a Virtual Agent," in Third International Conference on Games and Virtual Worlds for Serious Applications (VS-GAMES), 2011, pp. 164-167, doi: 10.1109/VS-GAMES.2011.31.

[20] C. Vassilakis, A. Antoniou, G. Lepouras, V. Poulopoulos, M. Wallace, S. Bampatzia, et al., "Stimulation of reflection and discussion in museum visits through the use of social media", Social Network Analysis and Mining, vol. 7, pp. 1-12, 2017, doi: 10.1007/s13278-017-0460-3.

[21] F. Bellotti, R. Berta, A. De Gloria, and V. Zappi, "Exploring gaming mechanisms to enhance knowledge acquisition in virtual worlds," in Proceedings of the 3rd international conference on Digital Interactive Media in Entertainment and Arts, Athens, Greece, 2008, pp. 77-84, doi: 10.1145/1413634.1413653.

[22] C. Brom, M. Preuss, and D. Klement, "Are educational computer micro-games engaging and effective for knowledge acquisition at high-schools? A quasiexperimental study", Computers \& Education, vol. 57, pp. 1971-1988, 2011, doi: 10.1016/j.compedu.2011.04.007.

[23] L. A. Annetta, J. Minogue, S. Y. Holmes, and M.-T. Cheng, "Investigating the impact of video games on high school students' engagement and learning about genetics", Computers \& Education, vol. 53, pp. 74-85, 2009, doi: 10.1016/j.compedu.2008.12.020.

[24] H. Lukosch, S. Kurapati, D. Groen, and A. Verbraeck, "Microgames for Situated Learning", Simulation \& Gaming, vol. 47, pp. 346-367, 2016, doi: $10.1177 / 1046878116635468$.

[25] CERN in Tripadvisor. Available: http://www.tripadvisor.co.uk/Attraction Reviewg188057-d242814-Reviews-CERN-Geneva.html

[26] A. Schaeffer. (2016) CERN tours: more popular than ever. Available: http://cds.cern.ch/record/2207255?!n=en

[27] CERN Media Lab. Available: http://medialab.web.cern.ch/

[28] A. Dix, "Human-Computer Interaction," in Encyclopedia of Database Systems, L. Liu and M. T. ÖZsu, Eds., ed Boston, MA: Springer US, 2009, pp. 1327-1331.

[29] Kinect for Windows. Available: https://developer.microsoft.com/enus/windows/kinect

[30] J. H. Brockmyer, C. M. Fox, K. A. Curtiss, E. McBroom, K. M. Burkhart, and J. N. Pidruzny, "The development of the Game Engagement Questionnaire: A measure of engagement in video game-playing", Journal of Experimental Social Psychology, vol. 45, pp. 624-634, 2009, doi: 10.1016/j.jesp.2009.02.016.

[31] A. I. Nordin, A. Denisova, and P. Cairns, "Too Many Questionnaires: Measuring Player Experience Whilst Playing Digital Games," presented at the The Seventh York Doctoral Symposium on Computer Science and Electronics, York, UK, 2014.

[32] M. K. Othman, H. Petrie, and C. Power, "Engaging Visitors in Museums with Technology: Scales for the Measurement of Visitor and Multimedia Guide Experience," Berlin, Heidelberg, 2011, pp. 92-99, doi.

[33] A. Corini, Z. Liu, W. Moore, and S. Osborn, "Evaluating Visitor Experience in the Citi Money Gallery at the British Museum", Faculty of the Worcester Polytechnic Institute, 2013.

[34] C. Jennett, A. L. Cox, P. Cairns, S. Dhoparee, A. Epps, T. Tijs, et al., "Measuring and defining the experience of immersion in games", International Journal of HumanComputer Studies, vol. 66, pp. 641-661, 2008, doi: 10.1016/j.ijhcs.2008.04.004.

[35] B. G. Consulting, "Evaluation of The Globe exhibition at CERN", Switzerland, January 2012.

[36] Wii. Available: http://wii.com/

[37] U. Ritterfeld, R. Weber, S. Fernandes, and P. Vorderer, "Think science!: entertainment education in interactive theaters", Computers in Entertainment, vol. 2, p. 11, 2004, doi: $10.1145 / 973801.973819$.

[38] HEAL Formal Evaluation Sample Questionnaire. Available: https://hcivr.dit.uop.gr/CERN_Game_Questionnaire.pdf 